\title{
Evaluation of pulmonary arterial pressure by Doppler colour flow mapping in patients with a ductus arteriosus
}

\author{
Kalimuddin Aziz, Habiba Tasneem
}

\begin{abstract}
Blood flow in the pulmonary artery was studied by Doppler colour flow mapping and cardiac catheterisation in 19 patients with a ductus arteriosus and different pulmonary artery pressures. In the four patients with normal pulmonary artery pressures colour Doppler flow mapping showed multicoloured wide and long systolic and diastolic jets in the pulmonary artery. In the 15 patients with raised pulmonary arterial pressure the systolic jets varied from multicoloured to red and were thinner: in patients with considerably raised pulmonary arterial pressure the jets became redder during diastole. The Doppler velocity tracings showed that in patients with normal pulmonary artery pressures the mean peak systolic velocity was higher than the mean end diastolic velocity-so that in all four the ratio of peak systolic velocity to end diastolic velocity was $<2$. The mean peak systolic velocity was much higher than the mean end diastolic velocity in 13 of the 15 patients with raised pulmonary artery pressure; this meant that the ratio of peak systolic velocity to end diastolic velocity was $>2$ in 10 of 11 patients. The end diastolic velocity was significantly lower in those patients with raised pulmonary artery pressure than in those with normal artery pressure. There was an inverse linear correlation between the mean pulmonary artery pressure and end diastolic ductal jet velocity in 17 of the 19 patients.
\end{abstract}

Colour flow mapping and this quantitative Doppler technique can detect pulmonary artery hypertension in patients with a ductus arteriosus.

The development of cross sectional echocardiography with pulsed and continuous wave Doppler techniques allows visualisation of the ductus arteriosus and recording of the flow velocity of ductal systolic and diastolic shunting in the pulmonary artery. A Doppler study showed that diastolic ductal flow was shortened in patients with pulmonary artery hypertension. ${ }^{1}$ There were significant correlations between the haemodynamically determined instantaneous aorta to pulmonary arterial systolic pressure gradient and maximum instantaneous Doppler velocity across the ductus and between the instantaneous aorta to pulmonary artery diastolic pressure difference and minimum instantaneous Doppler velocity across the ductus. ${ }^{2}$ The Doppler colour flow mapping technique not only images the ductus arteriosus but also characterises the systolic and diastolic jets in the pulmonary artery that are generated through the ductus. ${ }^{34}$ The Doppler imaging technique showed distinct retrograde blood flow patterns caused by left to right shunting in the main pulmonary artery in patients with a ductus arteriosus ${ }^{4}$ and a reduction in the diastolic ductal flow velocities when pulmonary arterial pressure was high. ${ }^{3}$ The purpose of our study was to define various abnormal patterns of blood flow in the pulmonary artery caused by a ductus of various sizes and to determine the relation between pulmonary artery pressure and diastolic and systolic ductal blood flow velocities recorded in the pulmonary artery by Doppler colour flow mapping.

\section{Patients and methods}

We studied 19 patients (mean (SD) age $4 \cdot 3$ (1.9) years; mean (SD) weight $14.3(3 \cdot 1) \mathrm{kg}$ ). Seventeen had a ductus arteriosus and two also had coarctation of the aorta. Patients were examined in the supine position and infants were sedated with chloral hydrate. The heart was imaged from all sites with a Toshiba colour flow Doppler echocardiogram (Sonolayer-SSH-65 A). The ductus arteriosus was imaged from the left parasternal oblique plane and from high left parasternal positions. ${ }^{56} \mathrm{We}$ used a continuous wave Doppler beam with concomitant imaging of the pulmonary artery and descending aorta. We took special care to record both the systolic and diastolic flow velocities within the main pulmonary artery. We used colour Doppler flow mapping to image the high velocity jets in the pulmonary artery and by aligning the Doppler beam parallel with the jet we recorded maximum velocity spectra. Audio signals were used to record the velocities in the laminar portion of the jet.

Cardiac catheterisation was undertaken within one week of the echocardiographic study. A mixture of pethidine, chlorpromazine, and promethazine hydrochloride was used as a premedication. Blood gas saturation and two to three pressures were measured from the right heart chambers and descending aorta. In patients with coarctation of the aorta pressure was recorded in the ascending aorta. The ratio of pulmonary blood flow to systemic 

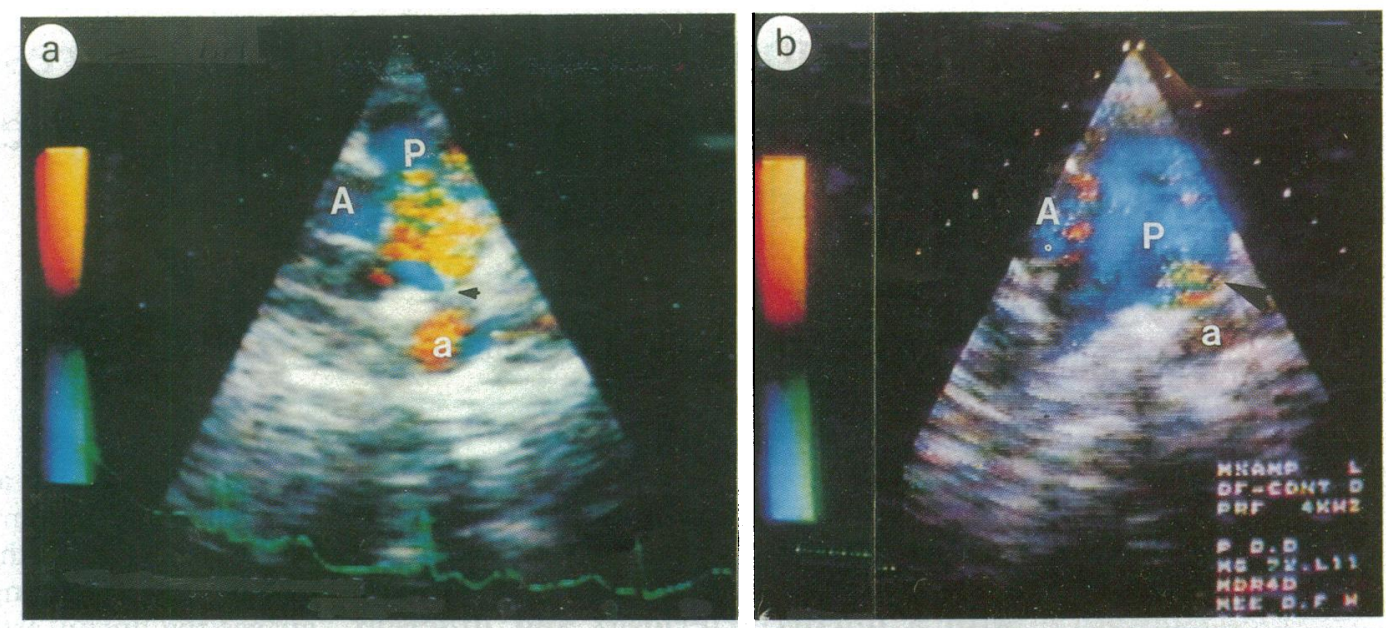

Figure 1 Colour flow mapping from the base of the heart in short axis plane from left parasternal location. (a) In a patient with a ductus arteriosus and normal pulmonary arterial pressure showing a multicoloured systolic jet arising from the ductus (arrow) and spreading into a wide area in the pulmonary artery $(P)$. Note an area of turbulence close to the ductus in the descending aorta.

$A$, ascending aorta; $a$, descending aorta. Short axis left parasternal colour flow mapping from the base of the heart in systole ( $b$ ) and diastole in a patient with a ductus arteriosus and pulmonary arterial hypertension, showing a multicoloured systolic jet coming from the ductus and extending into the main pulmonary artery $(P)$ for a short distance. During diastole (c) it became redder, suggesting considerably reduced diastolic jet velocities.

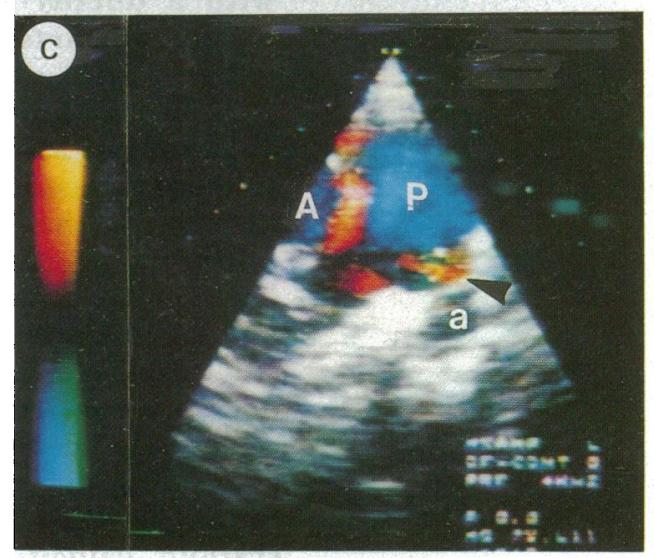

blood flow (QP/QS) was calculated for 17 of 19 patients. The peak systolic velocity was measured manually from the timed velocity spectra that were recorded on a hard copy. The highest point on the systolic velocity spectrum was taken as the peak systolic velocity measurement. Three to four measurements were taken and the mean value was used for the correlations. The end diastolic velocity was measured at the peak of the $R$ wave.

In one patient with considerably increased pulmonary artery pressure there was a drop out of diastolic velocities in the last part of diastole. In this patient we used the last portion of the diastolic spectrum to measure diastolic velocity. A mean of three to four measurements was taken. In four patients the systolic velocity spectrum was either not recorded or was incomplete. In two patients with significantly raised pulmonary arterial pressures, abnormal ductal flow velocities were not recorded in the pulmonary artery, in systole or diastole. These two patients showed no left to right shunt at cardiac catheterisation and one of them showed only a right to left shunt from the pulmonary artery into the descending aorta.

\section{Results}

QUALITATIVE STUDY

In patients with a ductus arteriosus the continuous wave record of the Doppler flow velocity profile of anterograde (normal) pulmonary blood flow (coded blue) was seen in the distal pulmonary arteries as a negative

velocity spectrum (below the baseline) and concomitant systolic jet velocities caused by ductal left to right shunt as retrograde blood flow toward the pulmonary valve were recorded as positive velocity spectrum (above the baseline). Positive blood velocities continued to be recorded during diastole. This retrograde ductal systolic and diastolic jet flow could be mapped in the colour mode, and a multicoloured systolic jet could often be traced from the descending aorta, where a small area of multicoloured flow disturbance was seen, through the ductus along the lateral and superior wall of the main pulmonary artery in a retrograde direction. The distance to which systolic and diastolic jets extended into the pulmonary artery varied; with low pulmonary artery pressure the systolic jet extended in a retrograde direction right up to the pulmonary valve and eventually ended up in a vortex near the pulmonary valve where it reversed to flow in an anterograde direction. A multicoloured high velocity jet with wide lateral flow velocities extending a long way into the pulmonary artery was characteristic of a ductus arteriosus and low pressure in the pulmonary artery. The jet remained visible during both systole and diastole and the colour profile of the jet during systole and diastole did not vary a great deal in patients with normal pulmonary arterial pressure (fig 1a). Anterograde pulmonary flow (coded blue) was seen in the distal pulmonary arteries. Reversed flow during diastole (coded red)that is towards the pulmonary valve-was seen near the bifurcation of the pulmonary arteries. $^{4}$ In those patients in whom pul- 

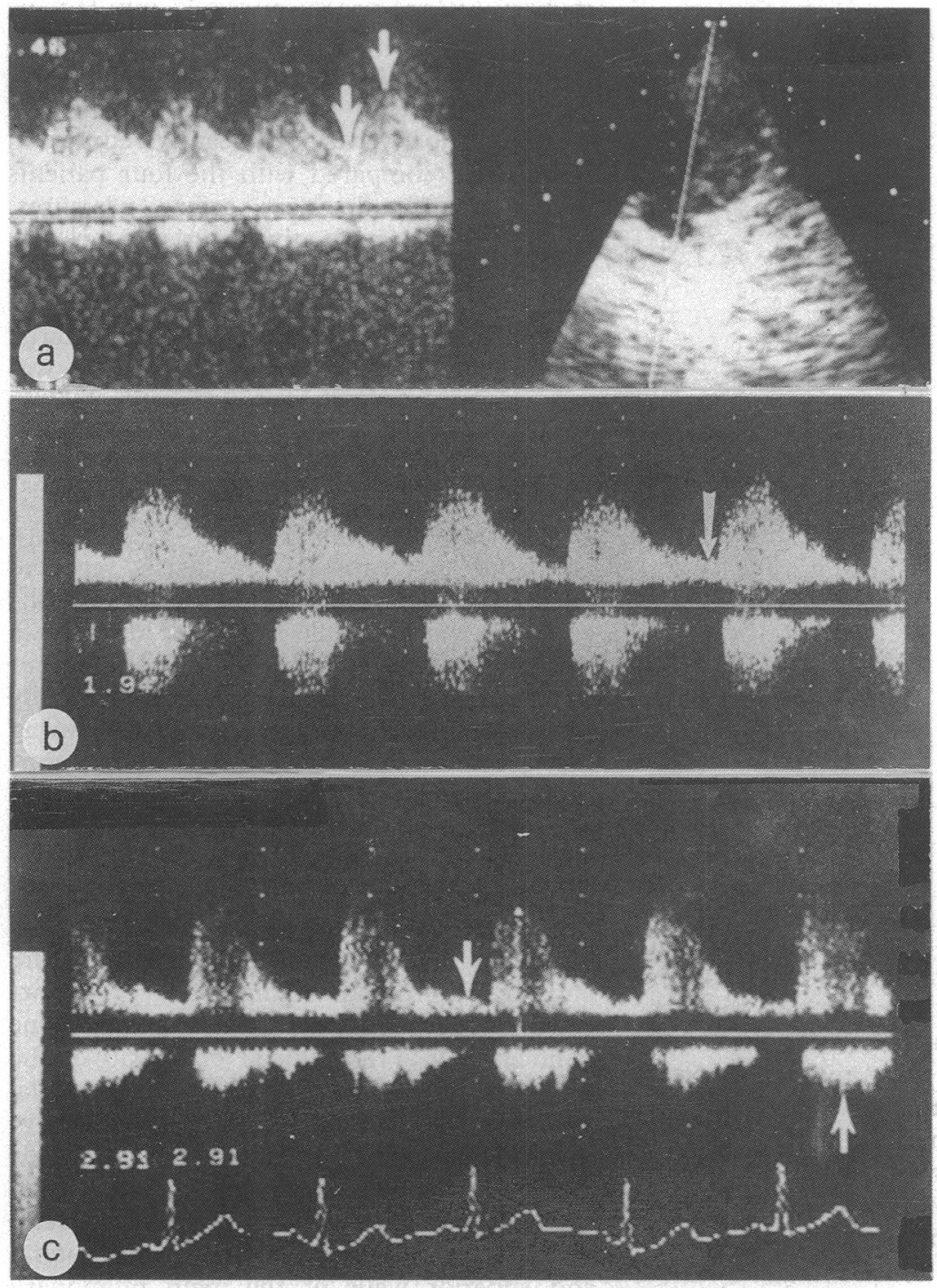

Figure 2 (a) Continuous wave Doppler velocity spectrum in the main pulmonary artery in a patient with a ductus arteriosus in whom the mean pulmonary artery pressure was $19 \mathrm{~mm} \mathrm{Hg.} \mathrm{Retrograde}$ flow velocities were continuously above the baseline during systole and diastole because of left to right ductal shunting. Both peak systolic (second arrow) and end diastolic velocities ( first arrow) were relatively high. (b) Continuous wave Doppler velocity spectrum recorded in the pulmonary artery in a patient with mean pulmonary arterial pressure of $59 \mathrm{~mm} \mathrm{Hg}$. Compared with peak systolic velocity the end diastolic velocity (arrow) was significantly reduced. Anterograde (normal) systolic pulmonary arterial flow velocities were recorded below the baseline. (c) Velocity spectrum of a patient with a ductus arteriosus and mean pulmonary arterial pressure of $62 \mathrm{~mm} \mathrm{Hg}$. The diastolic velocity was much reduced (arrow above the baseline). Arrow below the baseline shows anterograde (normal) flow velocities.

monary arterial pressure was considerably increased and the left to right shunt was small-that is the $\mathrm{QP} / \mathrm{QS}$ ratio was $<1.5-\mathrm{a}$ small flame shaped red jet came from the ductus and extended a short distance into the main pulmonary artery during systole and diastole. In the patients with raised pulmonary arterial pressure, the colour coding of the systolic multicoloured jet was variable and depended upon the pulmonary arterial pressure (fig $1 \mathrm{~b}$ ). In patients with moderately raised pulmonary arterial pressure the multicoloured systolic jet was shorter and thinner and became more red during diastole because of a considerable reduction in the diastolic velocities (fig lc). In one patient we did not see left to right ductal flow in the main pulmonary artery; however, the ductus image showed systolic and diastolic flow (coded blue) from the pulmonary artery into the

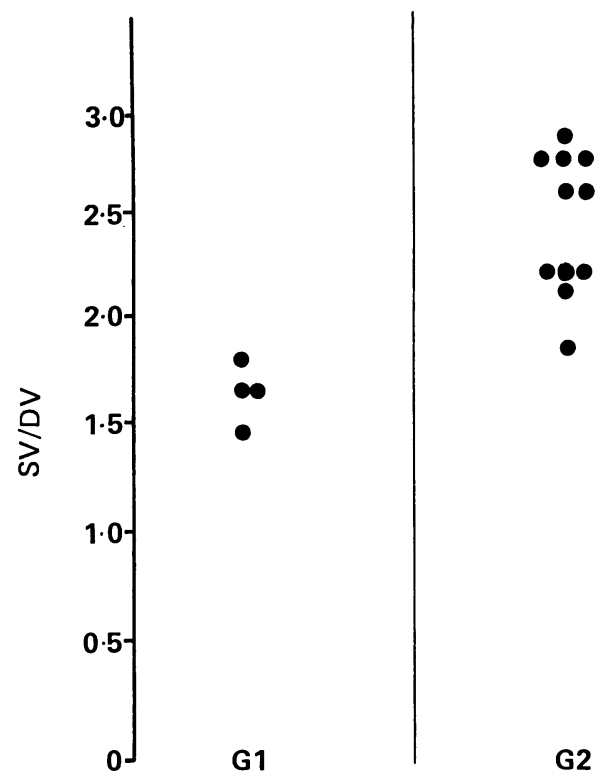

Figure 3 Peak systolic/end diastolic velocity ratio (SV) $D V$, in patients with low pulmonary artery pressures ( G1) and high pulmonary artery pressures (G2). All but one of the 11 hypertensive patients had ratios $>2$.

descending aorta. Catheter studies (oximetry) in this patient did not show shunting and pressures in the pulmonary artery and aorta were similar. The only abnormal velocities recorded in this patient were from reversed flow from the pulmonary artery through the pulmonary valve into the right ventricle, which were due to pulmonary regurgitation caused by very high pulmonary arterial pressure.

\section{QUANTITATIVE STUDY}

Left to right ductal shunting was studied by recording abnormal positive flow velocities (above the baseline) during systole and dia-

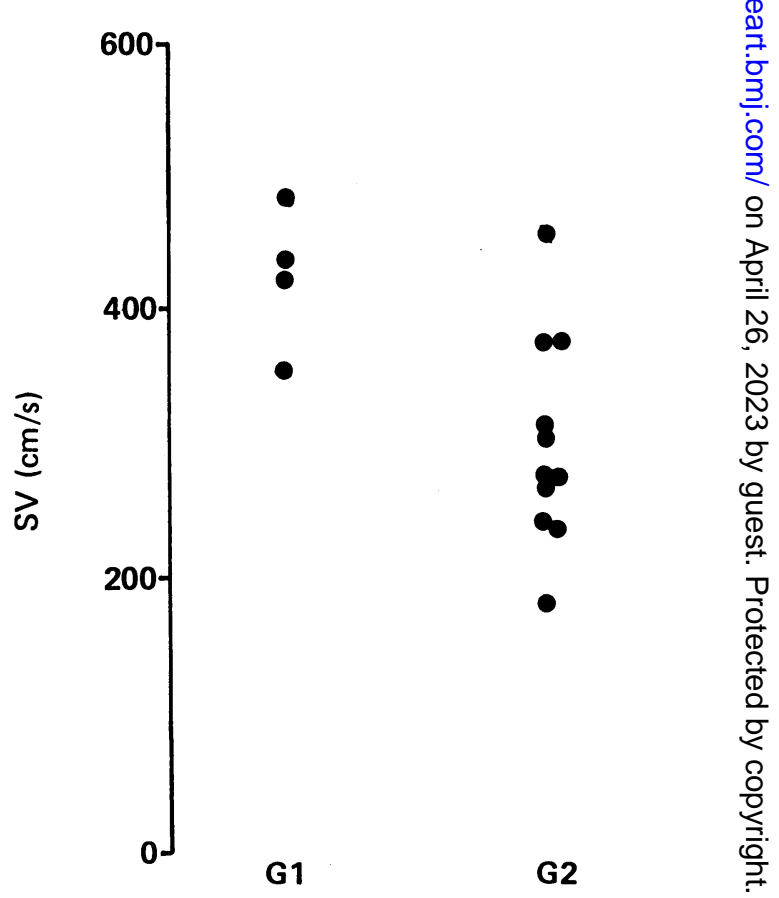

Figure 4 Peak systolic ( $S V \mathrm{~cm} / \mathrm{s})$ velocities in patients with low pulmonary artery pressures (G1) and high pulmonary artery pressures (G2). Peak systolic velocities were significantly lower in the hypertensive group $(p<0.01)$. 
Figure 5 End diastolic velocities $(D V \mathrm{~cm} / \mathrm{s})$ in patients with low pulmonary artery pressures (G1) and high pulmonary artery pressures (G2). There was no overlap of values in the normotensive and hypertensive groups $(p<0.001)$.

Figure 6 Mean pulmonary arterial pressure (MPAP) plotted against end diastolic velocity $(D V \mathrm{~cm} / \mathrm{s})$ in 17 patients with a ductus arteriosus. An increase in mean pulmonary artery pressure was associated with proportionate reduction in diastolic velocity.

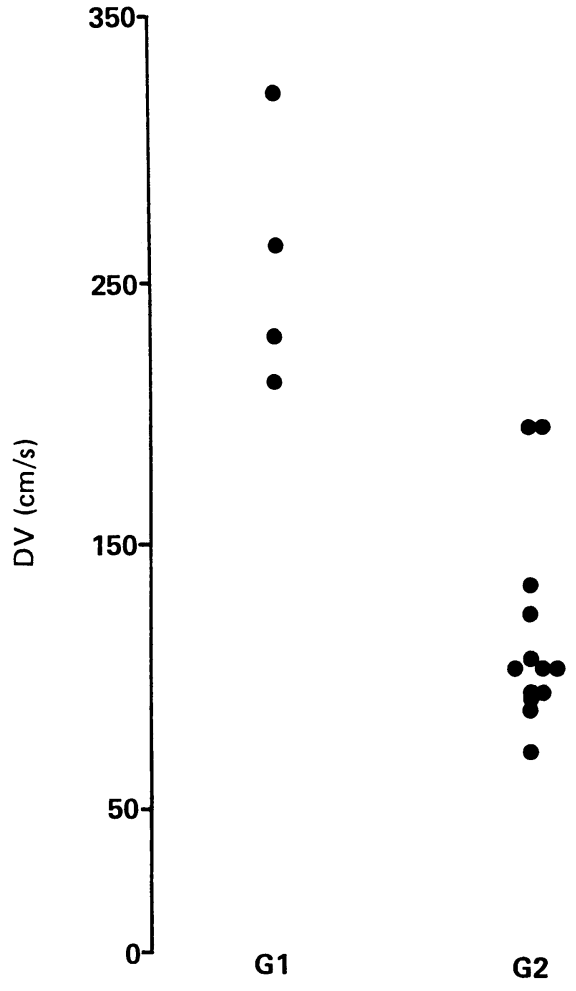

stole (fig 2). Cardiac catheterisation showed that in four patients (group I) mean pulmonary arterial pressure was $\leqslant 24 \mathrm{~mm} \mathrm{Hg}$ (mean (SD) $20.3(2.5) \mathrm{mm} \mathrm{Hg}$ ) and the mean (SD) $\mathrm{QP} / \mathrm{QS}$ ratio was $2 \cdot 1(0 \cdot 48)$ (fig $2 \mathrm{a})$. In 15 patients (group II) mean pulmonary artery pressure was $>25 \mathrm{~mm} \mathrm{Hg}$ (mean (SD) $55 \cdot 1$ $(11.7) \mathrm{mm} \mathrm{Hg}$ ). The mean $\mathrm{QP} / \mathrm{QS}$ ratio in 13 of these 15 patients was $3.4(1 \cdot 7$ ) (fig $2 \mathrm{~b}$ and c). The mean peak systolic velocity in the four patients with lower mean pulmonary arterial pressure was $434(52.9) \mathrm{cm} / \mathrm{s}$ and the mean end diastolic velocity was $264(50 \cdot 8) \mathrm{cm} / \mathrm{s}$ and the ratio of systolic velocity/end diastolic velocity was $<2$ (mean $1.68(0 \cdot 17)$. Mean peak systolic velocity $(308(78 \cdot 3) \mathrm{cm} / \mathrm{s})$ was measured in 11 of the 15 patients with higher pulmonary artery pressures (group II) and mean end diastolic velocity $(118.6(39.9) \mathrm{cm} / \mathrm{s})$ was measured in 13. The ratio of peak systolic velocity to end diastolic velocity was $>2$ (mean 2.5 (0.37)) in the 10 patients and in one patient the ratio was 1.9 and the mean pul-

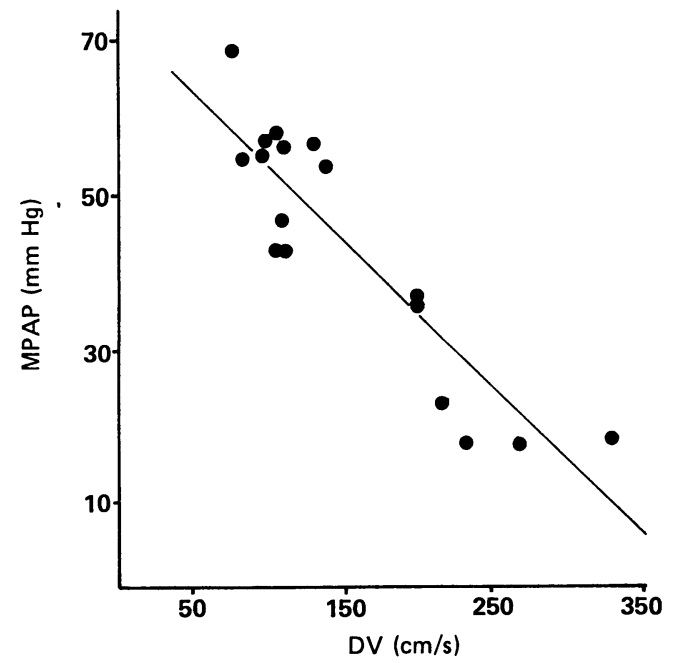

monary arterial pressure was $37 \mathrm{~mm} \mathrm{Hg}$ (fig 3 ). The peak systolic velocity was significantly lower in the patients with raised pulmonary artery pressures $(p<0.01$, fig 4$)$; however, diastolic velocity was significantly reduced in this group (compared with the four patients with normal pulmonary artery pressures) $(\mathrm{p}<0.001)$, and this variable clearly separated the two groups (fig 5).

The end diastolic velocity correlated inversely with the mean pulmonary arterial pressure in all 17 patients in whom it was measured $(\mathrm{r}=0.897, \mathrm{p}<0.001, \mathrm{SE} 0.0387)$ (fig 6).

\section{Discussion}

Our study confirms the previously reported colour Doppler profile of pulmonary systolic and diastolic blood flows in patients with a ductus arteriosus. ${ }^{4}$ Retrograde continuous flow toward the pulmonary valve caused by left to right ductal shunting was seen during systole and diastole. This retrograde flow contained velocities that depended upon the size of the ductus and the pulmonary arterial pressure during systole and diastole. The distance to which a jet arising from the ductus extended retrogradely into the pulmonary artery was a function of pulmonary arterial pressure and the anterograde hydraulic force of forward systolic pulmonary blood flow on one hand and left to right ductal jet velocity on the other. In patients in whom pulmonary arterial pressure was normal, the anterograde hydraulic forces opposing the systolic retrograde flow in the ductal shunt were lower so that the systolic jet was wide with considerable lateral flow velocities and tracked along the lateral and superior walls of the main pulmonary artery up to the level of the pulmonary valve where a large vortex was seen with ductal flow joining the anterograde pulmonary blood flow. The areas around the systolic jet were blue (showing normal forward pulmonary arterial blood flow). The distance that the systolic and diastolic jets extended from the ductus to the pulmonary valve was reduced in proportion to the pulmonary arterial pressure. When pulmonary arterial pressure was significantly raised by increased pulmonary vascular resistance, anterograde hydraulic forces were increased and the systolic ductal jet was lean and thin and was considerably deflected toward the walls of the main pulmonary artery. During diastole the jet became more red, suggesting a considerable reduction in diastolic velocities. In patients with pulmonary arterial pressure that was near systemic values a red flame shaped jet was seen throughout systole and diastole. This extended a short distance from the ductus into the pulmonary artery. The diastolic jet velocities of these patients were much reduced (to $<100$ $\mathrm{cm} / \mathrm{s}$ ).

Thus our study showed that the colour coding of the systolic and diastolic ductal jets, their width, and the distance they extended into the pulmonary artery can be used to evaluate the pulmonary arterial pressure in 
patients with a ductus arteriosus.

Doppler measurement of the ductal shunting flows showed that systolic and diastolic velocities were significantly higher in patients with low pulmonary arterial pressure-so that the ratio of peak systolic velocity to end diastolic velocity was $<2$. Compared with peak systolic velocities the end diastolic velocities were considerably reduced in the hypertensive group so that the ratio of peak systolic to end diastolic velocity was $>2$ in most patients. So the ratio of peak systolic velocity to end diastolic velocity could be used to identify patients with pulmonary arterial hypertension. The only difficulty with this approach was that the peak systolic velocity could not be recorded in four patients. However, the absolute diastolic velocity measurement separated all patients with pulmonary artery hypertension from those without.

Our initial data showed a linear relation between the mean pulmonary arterial pressure and the end diastolic velocity measurement. We believe this relation would have been even closer if it had been feasible to record the Doppler velocity spectrum simultaneously with the measurement of pulmonary arterial pressure at cardiac catheterisation.
Our study suggests that for clinical purposes mean pulmonary arterial pressure can be derived from the Doppler measurement of end diastolic velocity. The colour Doppler technique permits not only qualitative but also quantitative estimate of pulmonary arterial pressure in patients with a ductus arteriosus.

1 Stevenson JG, Kawabori I, Guntheroth WG. Non invasive detection of pulmonary hypertension in patent ductus arteriosus by pulsed Doppler echocardiography. Circulation 1979;60:355-9.

2 Musewe NH, Smallhorn JF, Benson LN, Burrows PE Freedom RM. Validation of Doppler derived pulmonary arterial pressure in patients with ductus arteriosus under different hemodynamic states. Circulation 1987; 76:1081-91

3 Hiraishi S, Horiguchi Y, Misawa $\mathrm{H}$, et al. Non invasive Doppler echocardiographic evaluation of shunt flow dynamics of the ductus arteriosus. Circulation 1987;75: 1146-53.

4 Liao PK, Su JW, Hung JS. Doppler echocardiographic flow characteristics of isolated patent ductus arteriosus. Bette delineation by Doppler colour flow mapping. $\mathrm{Am} \mathrm{J}$ Cardiol 1988;12:1285-91.

5 Smallhorn JF, Huhta JC, Anderson RH, Macartney FJ. Suprasternal cross-sectional echocardiography in assessment of patent ductus arteriosus. Br Heart $J$ 1982;48: ment of

6 Sahn DJ, Allen HD. Real time cross sectional echocardiographic imaging and measurement of the patent ductus arteriosus in infants and children. Circulation 1978; 58:343-54. 